\title{
Procesos de integración social de inmigrantes en escuelas de Huelva, España: diversidad cultural y retos educativos
}

\section{Social Integration Processes of Immigrants in Schools of Huelva, Spain: Cultural Diversity and Educational Challenges}

\begin{abstract}
Karen García-Yepes es Docente investigadora y Coordinadora Editorial en la Fundación Universitaria Juan de Castellanos (Colombia); estudiante de Doctorado en la Universidad de Huelva (España) (garciayepesk@ gmail.com) (http://orcid.org/0000-000 1-6433-2264)
\end{abstract}

Recibido: 2016-12-12 / Revisado: 2017-04-22 / Aceptado: 2017-05-16 / Publicado: 2017-07-01

\section{Resumen}

Este artículo surge como resultado de una Tesis de Maestría desarrollada en la Ciudad de Huelva (España), sobre procesos de integración social de inmigrantes en el entorno escolar. El objetivo consiste en analizar los procesos de integración de niños inmigrantes en las escuelas de Huelva, reconociendo el rol social que cumple la escuela con respecto a su formación y sus oportunidades de socialización. En cuanto a la metodología, se recurre al análisis del discurso a partir de datos obtenidos en entrevistas en profundidad y talleres de interculturalidad donde participaron estudiantes, docentes y padres de familia. Como principal resultado del análisis se plantea que el reto de la escuela no consiste en adaptar los contenidos curriculares a la diversidad existente en su entorno, sino que los debe gestionar efectivamente, atendiendo a las necesidades particulares de los inmigrantes. Pues allí, se ejercen derechos y deberes ciudadanos así como oportunidades de participación social. De esta manera se concluye que el reto de la escuela en contextos interculturales consiste en fomentar la integración social a través del desarrollo de habilidades para la vida y la socialización. Para ello, debe reorientar la trayectoria vital de niños que han dejado sus raíces, promoviendo un diálogo entre su pasado y su presente, permitiéndoles desarrollar estrategias concretas en favor de la construcción de su proyecto de vida en un nuevo contexto social.

Forma sugerida de citar: García-Yepes, Karen (2017). Procesos de integración social de inmigrantes en escuelas de Huelva, España. Alteridad, 12(2), 188-200. https://doi.org/10.17163/alt.v12n1.2017.05. 
Descriptores: Relaciones culturales, educación intercultural, integración social, inmigrantes, diversidad cultural, escuela.

\begin{abstract}
This article, focused on the social integration processes of immigrants in the school environment, derives as a result of a Master's Thesis developed in the city of Huelva (Spain). Its objective is to analyse the integration processes of immigrant children in the schools of Huelva, acknowledging the social role of schools regarding the children's formation and socialising opportunities. Methodology: discourse analysis applied to data gathered through in-depth interviews and workshops on interculturality with students, teachers and parents as participants. Main result of this analysis: the real challenge
\end{abstract}

\section{Introducción}

Debido a los recientes flujos migratorios hacia Europa desde Siria y otros contextos de conflicto, cabe preguntarse el papel que cumple la escuela en los procesos de integración social de los inmigrantes. En efecto, las relaciones en torno a la interculturalidad implican la creación de estrategias de conciliación por parte de las culturas presentes en una comunidad dados los conflictos derivados de la diversidad existente. Frente a este aspecto, resalta que la integración no solo es un mecanismo para promover la igualdad de trato y la inclusión social en el marco de esa diversidad, sino que también es una forma de promover la convivencia social.

De esta manera, el objetivo de este artículo consiste en analizar los procesos de integración entre los niños extranjeros en las escuelas de Huelva (España), reconociendo el rol social que cumple la escuela en su formación y teniendo en cuenta las repercusiones que implican las diferencias lingüísticas, los contrastes culturales o los procesos de socialización anteriores. Para este fin se analiza el papel que cumple la escuela como espacio integrador donde se construyen relaciones, redes de apoyo y diversas las formas de socialización. for the school is not to adapt curriculum's contents to the diversity of the environment, but to manage them effectively, meeting the particular needs of immigrants. It is there where citizens' rights and duties, as well as social participation opportunities are exercised. In this sense, it can be concluded that the challenge that schools face at intercultural contexts is to promote social integration through the development of abilities useful for life and for socialising. To this end, it is necessary to reorient the life course of children who left their roots, by promoting a dialog between their past and their present, which would allow the development of specific strategies contributing to the construction of their life course in a new social context.

Keywords: Cultural relations, intercultural education, social integration, immigrants, cultural diversity, school.

Es importante destacar que el concepto de "extranjeros" e "inmigrantes" se usa en este artículo para referirse a aquellos que no nacieron en España. En efecto, de acuerdo con el Código Civil español, se requiere tener al menos un padre español o habiendo nacido en España, no recibir la nacionalidad del país de origen al nacer, para adquirir la condición de nacional español (2002). Es decir, un niño nacido en España puede ser extranjero si no tiene esas condiciones. Así, se evidencia que la idea de "extranjero" también es una construcción cultural y simbólica que determina las posibilidades de integración social. Por último, es pertinente mencionar que desde la conceptualización teórica, "inmigrante" es la condición social de alguien que viene de otro país y "extranjero" es la condición administrativo-jurídica de una persona en un país (Colectivo Ioé, 2005).

Desde esta perspectiva resulta imprescindible reconocer la importancia de la educación como proceso que posibilita el derecho a la participación y el desarrollo integral. Efectivamente, es necesario adecuar el sistema educativo a la diversidad de procedencias culturales de los niños gestionando adecuadamente dicha complejidad y fomentando competencias interculturales entre el alumnado, las familias y los docentes. Desde esta 
perspectiva, impulsar el conocimiento y la comprensión de la diversidad cultural en el ámbito educativo permite mejorar los procesos de integración de los niños entre sí. En ese sentido, estas estrategias fortalecen la convivencia social ya que reconoce la inclusión como un proceso que depende de los niños y de las comunidades. Esto es lo que se analiza a continuación.

\section{Fundamentación teórica}

\subsection{De la educación asimilacionista a la educación intercultural: un camino hacia la integración social}

La realización de este trabajo se enmarca en los planteamientos teóricos aportados desde el concepto de educación intercultural. Este término es publicado por primera vez a finales de los años 60 por Jack Forbes en The education of the culturally different: a multicultural approach (Muñoz, 1997). Ante todo se trata de un concepto que hace referencia a la necesidad de crear un entorno de justicia social basado en los derechos y el respeto a los estilos de vida en el ámbito educativo teniendo como base la diversidad cultural.

Para realizar una mejor aproximación a este concepto es necesario tener como referencia el contexto de las reivindicaciones sociopolíticas de mediados de los años 60. Entre ellas se encuentran los movimientos afroamericanos en Estados Unidos y las diversas protestas juveniles en variados países de Europa. Se trata de demandas sociales y civiles que, desde luego tienen impacto en el ámbito educativo y consecuentemente, van ampliando el uso del término desde diversas disciplinas. Así mismo, la dinamización de los procesos migratorios en esta etapa del siglo XX da cabida a nuevas formas de comprender la educación como proceso destinado a mejorar las relaciones entre personas de diversas procedencias étnicas y culturales en el marco de unas relaciones sociales establecidas a partir de la igualdad y evitando así el segregacionismo. De acuerdo con Leurin (1987, citado por Aguado, 1991), este enfoque se basa en la posibilidad de prácticas educativas en las que se impulsa a los miembros de una sociedad a ser conscientes de su interdependencia.

Expuesta esta argumentación, queda claro que la interculturalidad en la práctica educativa puede entenderse como un proceso de diálogo en torno a la diferencia entendida como diversidad social y cultural pero en ningún caso como desigualdad. Es lograda al compartir el "ethos" y los puntos de vista del otra al interactuar entre sí (Aguado, 1991). Se pretende así deconstruir los antagonismos fundados en la idea de que solo las minorías étnicas son diferentes ya que, al contrario, todos los grupos, incluidos los mayoritarios, están construidos a partir de una identidad que los construye ante otros grupos. Según Banks, los programas académicos basados en la interculturalidad se pueden reconocer en aquellos destinados a mejorar el rendimiento académico así como la integración social de dichas minorías y en la misma medida, intentan mejorar los procesos comunicativos entre ambas culturas para generar un diálogo intercultural constructivo (1989).

En otras palabras, se quiere enfatizar que la educación intercultural implica el encuentro de toda la sociedad en el marco de un entorno común de intercambio cultural y no exclusivamente a las minorías étnicas e inmigrantes. Por ello, es posible argumentar que no basta con constatar la diferencia sino que en sí misma esta es enriquecedora (Vázquez, 2001). Ya a principios de los años 80, en Dublín, la Conferencia de Ministros de Educación en el Consejo de Europa se pronunciaba en favor de programas educativos diseñados para integrar en forma dinámica las contribuciones culturales de los migrantes en los diferentes países del continente, con el objetivo de alcanzar una dimensión intercultural en educación (Consejo de Europa, 1983).

No obstante, la dificultad surge cuando los modelos académicos y las instituciones sociales, no abordan adecuadamente las diferencias. Efectivamente, a pesar de observar que los discursos oficiales e incluso, las políticas de Europa y España en materia de educación se orientan hacia la interculturalidad, aún se hace evidente que otros 
planteamientos dificultan la gestión de la diversidad. Ya sea de manera consciente o simplemente por las dificultades que implican el adaptar los programas curriculares al entorno intercultural en las aulas, se tiende a desarrollar procesos de enseñanza vinculados a los modelos culturales imperantes lo cual dificulta la integración de las minorías y los inmigrantes. Además, aún existe falta de concienciación social sobre este proceso. Con relación a esta idea por ejemplo, Sartori considera que las estrategias interculturales son causantes de conflictos porque en realidad la política del reconocimiento multiplicaría las diferencias (2001).

De esa forma, estas últimas propuestas estarían más acordes con las alternativas asimilacionistas o compensatorias que con otras formulaciones educativas vinculadas a la interculturalidad y más cercanas a la educación inclusiva, global o multicultural (Aguado, 2005). Con respecto a esto, la educación asimilatoria promueve la adaptación del alumnado a la cultura hegemónica (Dietz, 2003) Es decir, se procura homogeneizar la diversidad cultural existente en la escuela. Por su parte, la educación compensatoria busca superar dificultades de adaptación cultural y lingüística entre alumnos de grupos culturales minoritarios (Dietz, 2003). Por tanto, a la larga también se busca el mismo objetivo que en el caso anterior. La dificultad en esos discursos reside en que tienden en traducir la diversidad en desigualdad y remarcan los contrastes entre las culturas, reduciendo la identidad del individuo al resultado de una procedencia étnica determinada. En este sentido, cabe afirmar que las diferencias no se deben establecer entre grupos étnicos sino entre individuos mediatizados por circunstancias culturales así como individuales (Vázquez, 2001). No es posible obviar el hecho de que aparte de unas tradiciones culturales, también existen unos procesos de socialización y una identidad particular que igualmente determinan las formas según las cuales se relaciona el individuo en la sociedad.

En este sentido, "integrar" no debe equiparse con la asimilación de las prácticas educativas de la sociedad de acogida ni a resaltar los con- trastes entre los niños. En el caso específico de los niños inmigrantes, ello conlleva a potenciar las dificultades que supone un proceso de migración cuyas repercusiones implican una reacomodación de la identidad personal y cultural. Esto es evidente porque esa situación en la que se confunde la integración con la adaptación, es la que influye sobre la construcción de las ideologías y las actitudes que reproducen los conflictos (Carbonell, 1999). Dada esta situación, las prácticas escolares que buscan la incorporación de los niños a partir de la segregación para "compensar", tenderían a reproducir las diferencias como "desigualdades". Por el contrario, desarrollar procesos más inclusivos en el ámbito académico y tener en cuenta que la educación intercultural implica a todos los actores escolares, facilitaría la comprensión del otro y la reflexión sobre sí mismo al margen de la mirada etnocentrista de la cultura. De ello, se desprende que la educación para la integración social debe promover el encuentro y el diálogo de los distintos grupos sociales que conviven en la escuela, la construcción de aprendizajes colectivos y la preservación de su propia identidad (Rodríguez, 2004). Por su parte, la educación para la inclusión abarca un foco más amplio y hace referencia a la posibilidad de garantizar el acceso, la participación y el éxito de todos los estudiantes, especialmente aquellos que pertenecen a grupos sociales o culturales minoritarios (Blanco, 2006).

\subsection{Procedencia cultural y procesos de socialización}

La cultura es una representación de símbolos socialmente establecidos que adquieren significado en medio de un contexto particular. A partir de esta argumentación, se percibe que los individuos se encuentran mediatizados por esas configuraciones enraizadas en la conciencia colectiva, pero aparte de eso, existe un margen de individualidad que configura a los individuos. Esta afirmación tiene que ver con el desarrollo de la identidad siendo un proceso mediatizado por factores que no solo responden a explicaciones culturales. En 
esa medida, otras categorías específicas relacionadas con la construcción del género, la familia, las clases sociales, la vida académica, el trabajo o las relaciones interpersonales, definirían la construcción de la individualidad. En otras palabras, las personas se definen a sí mismas en el seno de esas interdependencias recíprocas construidas a partir de las configuraciones sociales a las que pertenece (Chartier, 1983).

Específicamente, se hace referencia al hecho de que a pesar de pertenecer a una misma cultura, las personas responden a circunstancias particulares en torno a las relaciones familiares o sociales. Desde ese punto de vista, se configuran diferentes procesos de socialización entendidos, en ese caso, como formas de construir la identidad y de desarrollar actitudes en torno a diversos aspectos de la vida social.

En efecto, al considerar las formas en las que los niños se integran en el contexto educativo, hace falta afirmar que su condición de "migrante" no implica necesariamente dificultades para estudiar o relacionarse. Por el contrario, se trata de niños con trayectorias de vida particulares que manifiestan necesidades concretas. Se vincula sobre todo con las dificultades que comporta la readaptación a una nueva lengua, a una nueva cultura o a una nueva escuela al responder a valores distintos a los aprendidos desde la familia, o en la vida cotidiana y ya no desde el concepto global de "cultura". En resumen, los procesos de socialización en la escuela también están orientados por este tipo de referentes sociales (Díaz, Franco, Martínez \& Pozo, 2001).

$\mathrm{Si}$ bien es cierto que las concepciones en torno a la cultura y el origen de los niños, tienen repercusiones en sus formas de socializar y de relacionarse, también es cierto que cada uno de ellos desarrolla una personalidad que adquiere matices distintos ante el rechazo, la aceptación o la integración. Por ello, no es posible observarlos a través del estereotipo que conlleva determinada procedencia cultural o regional. Es necesario ubicar su complejidad particular, social, cultural y económica teniendo en cuenta que no siempre la cultura determina los procesos particulares (Díaz \& otros, 2001).

\section{Metodología}

\subsection{Caracterización de la población}

La investigación se realizó con 53 niños pertenecientes al "Aula Promociona"1 de Fundación Secretariado Gitano ${ }^{2}$. De ellos, cinco provienen del extranjero: dos de Ecuador, uno de Chile, uno de Colombia y uno de Rumanía. Sin embargo, en las aulas y entre los niños no pertenecientes al programa, hay otros de nacionalidad marroquí, rumana, ecuatoriana, china, y colombiana. De los pertenecientes a Promociona, 18 se encuentran entre $5^{\circ} \mathrm{y}$ $6^{\circ}$ de Educación Primaria ${ }^{3}$ y tienen entre 11 y 12 años. Los restantes 35 niños están entre $1^{\circ}$ y $4^{\circ}$ de Educación Secundaria 4 con edades entre los 13 y 16 años; es decir, el $66,1 \%$ de los niños pertenecientes al programa. Se trata de niños de escuelas ubicadas en los Distritos III y V de Huelva:

- Distrito III: barriadas de Navidad, Santa Lucía, Cardeñas, La Gavilla, Las Colonias, El Carmen y Hotel Suárez.

- Distrito V: barriadas del Torrejón, Verde Luz, Hispanidad y Tres Ventanas.

En cuanto a las escuelas hay algunas que están adscritas oficialmente al programa y otras que son intervenidas de manera ocasional. De esa manera, esta investigación se realizó con los niños pertenecientes a las siguientes:

- Vinculadas por convenio con el Aula Promociona: CEIP Onuba, CEIP Andalucía, IES Virgen de Belén (Cinta), IES Virgen de Belén (Barriada de Navidad), IES La Marisma, CEIP Manuel Siurot, Colegio Hispanidad.

- Intervenidas ocasionalmente: Colegio Safa Funcadia, Colegio Cardenal Spinola, CEIP José Oliva, IES Los Rosales, Ciudad de los niños.

A continuación, se presentan cuatro gráficas donde se caracteriza la población por edades, cursos, sexo, total de estudiantes y escuelas. 


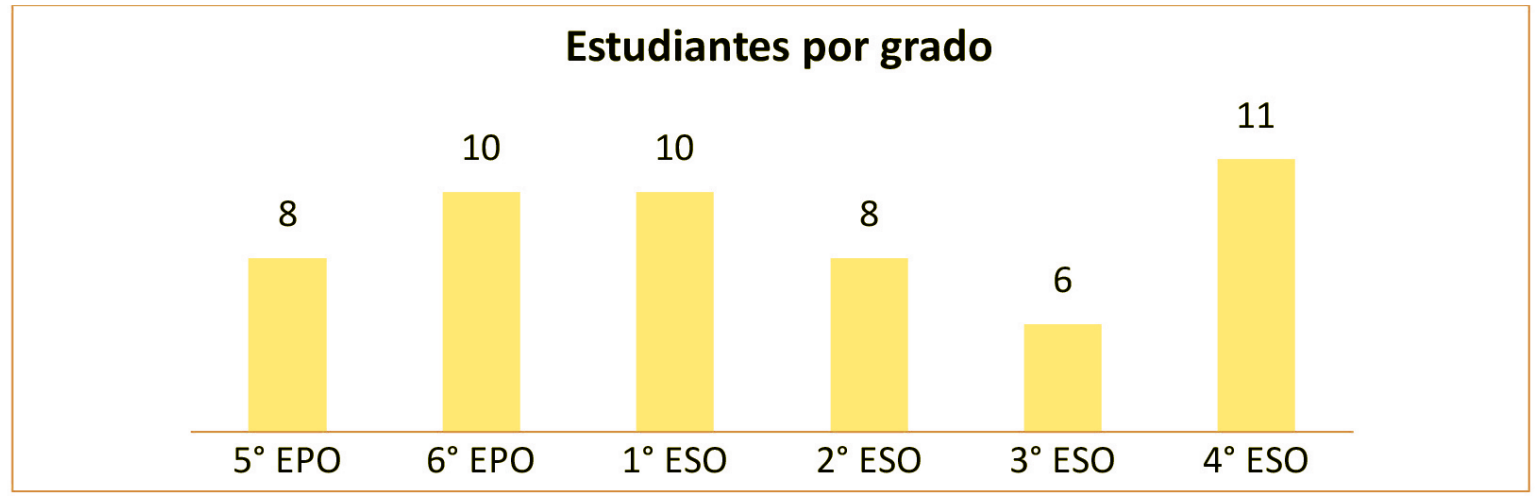

Fuente: elaboración propia

Gráfica 2

\section{Estudiantes por sexo}

31

22

Niños

Niñas

Fuente: elaboración propia

\section{Gráfica 3}

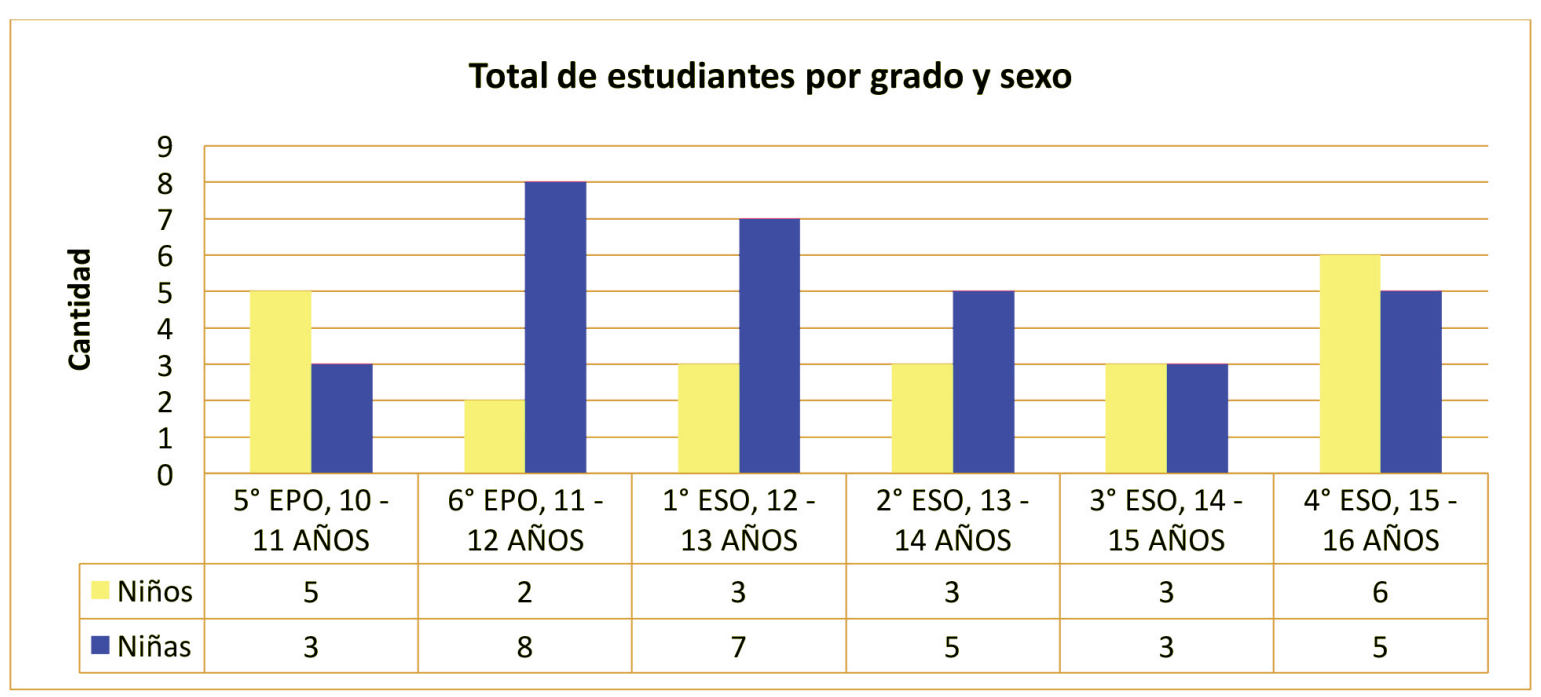

Fuente: elaboración propia 


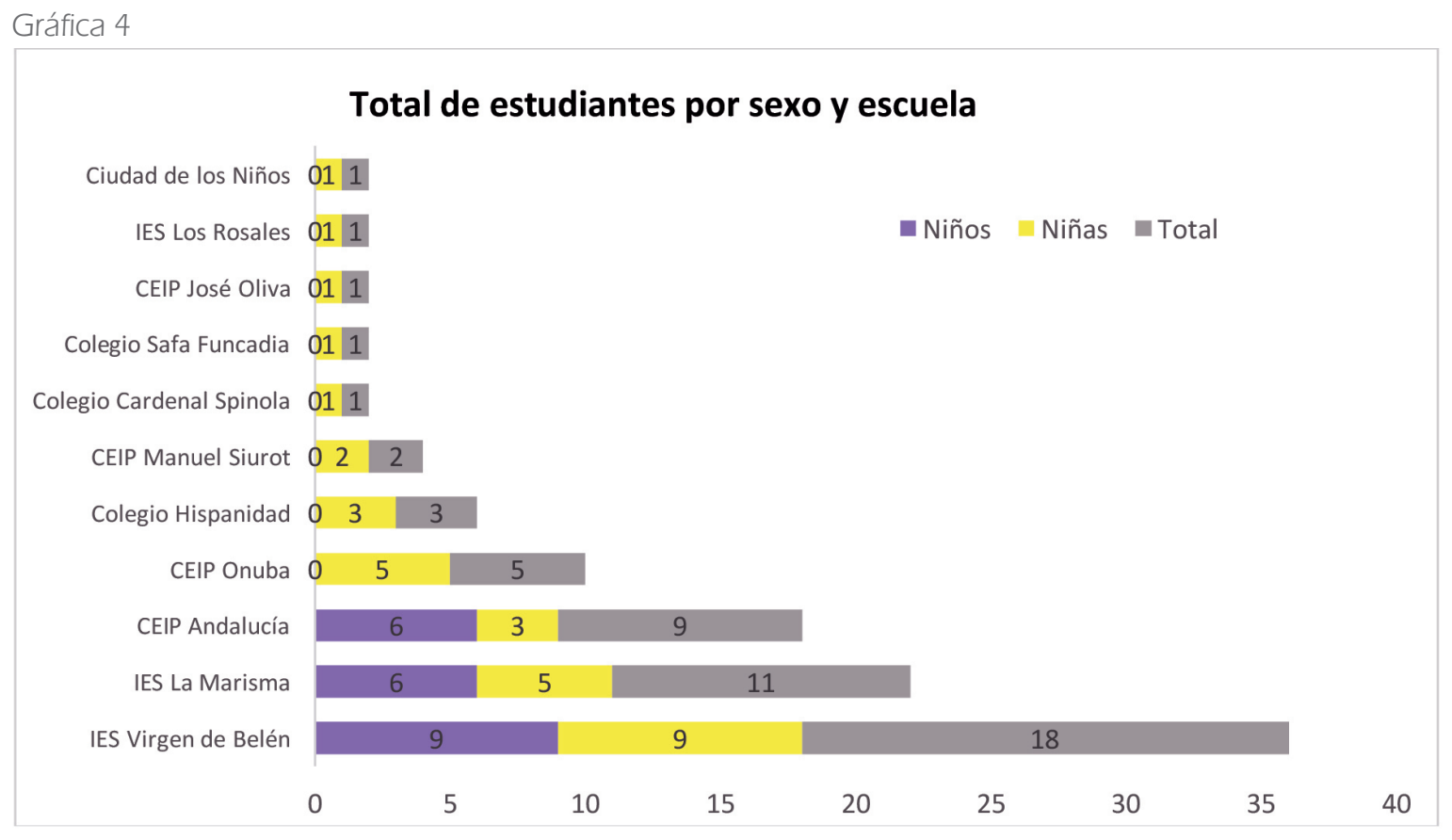

Fuente: elaboración propia

\subsection{Descripción del proceso metodológico}

\subsubsection{Enfoque metodológico}

Se utilizó el análisis del discurso como enfoque metodológico que sirve para comprender las prácticas discursivas que se producen en un contexto social donde la palabra oral y escrita forma parte de las actividades que allí se desarrollan (Calsamiglia y Tusón, 1999). El objetivo de esta estrategia está vinculado con la necesidad de conocer el sentido que los actores relacionados le atribuyen a las situaciones planteadas. Para ello, se utiliza un razonamiento de tipo inductivo, donde se realizan algunas conclusiones a partir de ejemplos particulares (Dávila, 2006). Así mismo, se espera que esto sea una manera propicia para conocer valores, formas de pensar y reacciones frente a la diversidad. Con ello, se pretende conocer los comportamientos particulares en torno a las relaciones interculturales en el aula con el fin de comprender los procesos de socialización de los niños y sus formas de integración social. Así, se abordan tres temáticas principales: las representaciones sociales, los procesos de socialización y las dinámicas de enseñanza - aprendizaje.

\subsection{Fases del proceso investigativo}

\subsubsection{Primera fase: Recolección de datos}

Para la recolección de datos se recurrió a talleres de educación intercultural y entrevistas en profundidad con los niños extranjeros, docentes y padres de familia. Así, se hicieron preguntas para caracterizar a la población e identificar actitudes con respecto a las relaciones interculturales en la escuela.

\subsubsection{Segunda fase: Sistematización de la infor- mación y análisis de los datos}

Posteriormente, se sistematizó la caracterización de la población. Luego, a partir de la clasificación de los discursos aportados por niños, docentes y padres de familia, se comenzó a construir una 
matriz de análisis para identificar los principales hallazgos. De ahí, surgieron tres categorías y cuatro subcategorías por cada una. Además, esto sirvió para obtener un análisis preliminar de la información. Todo se sintetiza en la siguiente matriz de análisis.

Tabla 2

\begin{tabular}{|c|c|}
\hline & Matriz de análisis \\
\hline Categoría & Subcategoría \\
\hline \multirow{4}{*}{$\begin{array}{l}\text { Representaciones sociales, } \\
\text { prejuicios y estereotipos }\end{array}$} & Representaciones culturales \\
\hline & Actitudes de los compañeros (rechazo, aceptación, comprensión) \\
\hline & Asimilacionismo y educación compensatoria \\
\hline & Educación intercultural \\
\hline \multirow{4}{*}{$\begin{array}{l}\text { Procesos de socialización y } \\
\text { procedencia cultural }\end{array}$} & Apoyo familiar \\
\hline & Conceptos sobre el papel de los padres y los educadores \\
\hline & Tradiciones, religión y cultura \\
\hline & Proceso de socialización, identidad e individualidad. \\
\hline $\begin{array}{l}\text { Estructuras curriculares } \\
\text { y entorno académico }\end{array}$ & Programas curriculares que no responden a la diversidad \\
\hline
\end{tabular}

Fuente: elaboración propia

\section{Análisis y resultados}

\subsection{Factores que influyen en los pro- cesos de integración social de los niños extranjeros en Huelva (España)}

El desarrollo de las temáticas planteadas tiene la intención de conocer las actitudes y conductas concretas que influyen en la integración social de los niños en las aulas. Específicamente este apartado se ha divido en tres partes fundamentales: la primera es sobre las representaciones sociales, los prejuicios y los estereotipos; la segunda es sobre los procesos de socialización y la procedencia cultural; y, la tercera, está relacionada con las estructuras curriculares y el entorno académico.

Así, se considera necesario conocer los aspectos relacionados con las percepciones de profesores y niños en torno a los extranjeros y viceversa. En segundo lugar, se estudian los refe- rentes culturales y la propia individualidad de los niños, a partir de un análisis desde el entorno familiar y sus expectativas ante el futuro. Por último, se analizan las actitudes relacionadas con los comportamientos frente los procesos educativos desarrollados en las aulas y las carencias detectadas en estos procesos.

Para este fin, se pretenden analizar los discursos de los niños, profesores y padres ante las situaciones que se plantearon en el transcurso de las actividades desarrolladas. Así, este trabajo recoge diferentes opiniones e historias relacionadas con el fin de ilustrar sus percepciones y fundamentar la argumentación desarrollada a continuación.

\subsection{Representaciones sociales, prejui- cios y estereotipos}

$\mathrm{Al}$ analizar las escuelas en la ciudad de Huelva, se hace evidente que las relaciones entre los niños inmigrantes y autóctonos, están mediadas por la complejidad que comportan sus visiones particulares frente a la cultura del "otro". Subyacen de 
fondo las construcciones sociales que a sus $10 \mathrm{y}$ 11 años, los niños han logrado construir a través de sus procesos de socialización, sobre los distintos grupos que cohabitan el contexto español. Sin embargo, es preciso aclarar que dos actitudes confluyen entre sí de manera recíproca. Por un lado, es evidente que entre los niños estos imaginarios sobre otros grupos sociales posiblemente ayudan a establecer la visión sobre uno solo de ellos. Por otro, estas actitudes no necesariamente son determinantes en los procesos de integración establecidos.

En principio, los imaginarios colectivos son aquellos sistemas simbólicos mediante los cuales una sociedad se construye con respecto a otra y crea una identidad social que dimensiona una manera particular de interrelacionarse. Como resultado de un proceso histórico que ha legado una memoria colectiva en una organización social, también es un elemento cohesionador y funciona como base de la estructura cultural e ideológica de la sociedad, elementos que constituyen su autorepresentación. De acuerdo con Baczko:

Estas representaciones de la realidad social (y no simples reflejos de ésta), inventadas y elaboradas con materiales tomados del caudal simbólico, tienen una realidad específica que reside en su misma existencia, en su impacto variable sobre las mentalidades y los comportamientos colectivos, en las múltiples funciones que ejercen en la vida social (1999).

Así, las representaciones colectivas tienen un efecto legitimador de ideologías y símbolos socialmente establecidos que adquieren significación dentro de una sociedad particular.

\subsection{El alumnado extranjero: Entre la igualdad, la adaptación y la interculturalidad}

Con respecto a la situación de los alumnos extranjeros en las escuelas visitadas en Huelva, se pudo detectar la existencia de tres discursos principales:
El de la igualdad, el de la educación compensatoria y el de la adaptación mutua.

El discurso de la igualdad destaca la necesidad de establecer una equiparación en los derechos y oportunidades de todos los alumnos. Paradójicamente, tiende a controvertir la esencia de la diversidad cultural, manifestando que esa similitud de posibilidades, también implicaría usar las mismas estrategias en cuanto a la educación de los niños inmigrantes. Principalmente, se trata del discurso asimilacionista donde se reconocería que las posibilidades de integración social de los niños están directamente relacionadas con su acomodación al contexto cultural subyacente.

Cierto es que discursos como este destacan al resaltar la igualdad en los derechos entre los alumnos al ser escolarizados, pero también es cierto que obvian la dimensión socio - cultural e individual del niño al sugerir que se requiere un proceso de adaptación a la sociedad de acogida en vez de ser un proceso mutuo. De otro lado, discursos similares enfatizan sobre las posibles repercusiones a nivel escolar dadas las dificultades que comporta el proceso migratorio.

El discurso de la pedagogía compensatoria por su parte, reconoce la diversidad presente entre los niños, tanto en los aspectos referidos a su procedencia cultural como a su situación social o familiar e incluso sus particularidades individuales. Sin embargo, se orienta a estrategias que compensan los aspectos académicos del proceso de integración. En última instancia, también se buscaría la "adaptación" a la cultura autóctona. Sobre todo, está relacionado con apreciaciones sobre ciertas dificultades académicas que tendría el niño como consecuencia del proceso migratorio.

Desde luego, este ideario reconoce la complejidad que puede comportar el niño pero se enfoca en las posibles metodologías a corregir si este pretende lograr mejores resultados académicos. A veces se encauza en aspectos específicos, tales como mejorar la enseñanza del castellano a los alumnos marroquíes o chinos. De otro lado, también reconoce que los niños latinoamericanos 
podrían tener menores dificultades al compartir una lengua común. Es decir, al contrario de lo que se espera, se podría dificultar el proceso de aprendizaje al fundar expectativas desarrolladas en torno a ciertos estereotipos creados a partir de la condición de alumno inmigrante.

Finalmente, el último discurso promueve la idea de la adaptación mutua, que no de la interculturalidad. En efecto, otros profesores se muestran de acuerdo con la necesidad de adaptar las propuestas pedagógicas a la diversidad cultural presente en las aulas, pero argumentan que en muchas ocasiones genera dificultad. En general, se afirma que hace falta adaptar las estructuras curriculares para desarrollar nuevas relaciones entre los niños en el marco de un proceso de integración recíproco, donde se reconozca a cada grupo cultural como diferente y valioso en sí mismo.

\subsection{Relaciones entre niños extranjeros y autóctonos}

A pesar de tener entre 10 y 11 años, los niños se reconocen como agentes activos del proceso migratorio y no solo como hijos e hijas de extranjeros recién llegados al país. Ellos también se sienten como actores fundamentales en ese proceso de reestructuración familiar y social porque saben que al igual que sus padres, dejaron sus amigos, sus familiares y sus escuelas. Así, se puede ver en los niños de esta edad que ellos están reconstruyendo su identidad a partir de un proceso continuo de interrelaciones entre la sociedad que dejaron y la sociedad de acogida. De este modo, algunos de los niños que llegaron siendo más jóvenes y tienen una tendencia más clara hacia la asimilación cultural, aceptan que también pertenecen a ese "otro" país que conocen a través de sus padres.

Por ejemplo, un chico nació en el mismo país que su madre y en su discurso, como en el de otros niños, se manifiesta una tensión constante entre ese país que anhelan y aquél donde han construido sus redes sociales desde la niñez o donde están redefiniendo su identidad. De hecho, al iniciar la adolescencia, están desarrollando ese proceso todavía. De otro lado, es evidente que esa circunstancia está condicionada por los niveles de aceptación o rechazo que manifiesta el entorno escolar (Cabrera, Marín, Espín, Rodríguez y, 1998). Así, algunos niños reconocen la realidad de las dificultades derivadas del proceso de integración en la nueva sociedad.

El otro discurso desarrollado de manera habitual por los niños es de la adaptación mutua. De hecho, suele afirmarse a través de estas argumentaciones que siempre el principio conlleva ciertas dificultades pero posteriormente se logran sortear dado que los niños autóctonos en última instancia, toman diversas actitudes que van desde la aceptación hacia la tolerancia, el respeto o la amabilidad. En efecto, uno de los hechos que resalta es que los estereotipos, los prejuicios o las posibles reservas frente a un niño de otra cultura o nacionalidad, se anulan en cierta medida cuando la convivencia escolar permite individualizar a los niños. Es decir, se sustraen los individuos de las construcciones sociales frente a determinados colectivos al reconocer sus particularidades sin perjuicio de que los estereotipos frente a otros miembros de esos colectivos se puedan mantener. Aunque es cierto que en ocasiones, estas diferencias culturales no definen criterios según los cuales los niños autóctonos pretendan escoger a sus amistades.

\subsection{El papel de la familia y la proce- dencia cultural}

Con respecto a los referentes culturales de los niños, el menor o mayor apoyo que reciban desde la familia así como los valores inculcados desde el hogar, son algunos de los factores que influyen en esos procesos de socialización. En la investigación realizada en las escuelas de la ciudad de Huelva se detectó la presencia de tres discursos principales en torno a la influencia familiar en la integración de los niños: el de la escuela como beneficio, el de los padres como educadores y el de la ausencia familiar.

En el primero de ellos los padres reconocen el papel de la escuela como factor decisivo en el desarrollo de sus hijos. Se produce una inte- 
rrelación entre sus frustraciones al no terminar los estudios secundarios y la aspiración de que se logren sus aspiraciones a través de los niños. En ese sentido, los espacios académicos cumplirían una función social en la medida en que sirve como instrumento para tener oportunidades sociales. Se produce así una relación directa entre el proceso de exclusión a causa de su no escolarización y la posibilidad concreta de acceder al mundo laboral.

El segundo hace referencia al cuestionamiento que hacen los padres sobre el papel de los profesores en la educación de los niños. En ocasiones, se trata de la idea según la cual los padres consideran que son quienes deben educar a los niños. De otro lado, se produce una tensión entre dos visiones distintas del espacio conocido como "escuela". Por una parte se trata de la visión sobre el hogar como escenario de educación integral, donde sus hijos adquieren valores requeridos para convivir en sociedad. Y de otra parte, también se trata de la concepción de estos padres sobre las falencias de la educación occidental, donde no sería posible aprender lo que ellos enseñan en el hogar.

El tercero se relaciona con la perspectiva de los profesores ante las repercusiones derivadas de la poca participación de los padres en los procesos educativos. Efectivamente, a lo largo de los programas de motivación a los que fueron invitados, solo muy poco de ellos acompañaron a sus hijos. En este sentido, los educadores sugieren que algunos padres no siempre están disponibles para cooperar con su formación, situación que se pudo comprobar. Sin embargo, resulta paradójico que las actividades desarrolladas en el marco del "Aula Promociona" resultan de una inscripción voluntaria al programa. De cualquier manera, esta aclaración adquiere relevancia cuando sirve para evidenciar que hay una tensión constante entre el deseo de lograr el éxito de los hijos y la idea sobre las formas en los que ellos podrían colaborar con este proceso.

\subsection{Estructuras curriculares y entorno académico}

También se ha encontrado que las dificultades escolares entre algunos niños extranjeros tienen que ver con las diferentes formas de comprender el conocimiento. Es evidente que las formas de aprendizaje cambian de una cultura a otra y en el caso de las aulas, esto se manifiesta con mayor intensidad. En este caso, el problema reside en que los programas curriculares son insuficientes para atender a la diversidad cultural de los niños y ello suele generar desmotivación entre los educadores. Además, esta situación es la que ocasiona las dificultades académicas entre algunos menores, dado que algunas de esas estructuras curriculares son ajenas a sus intereses.

Por ello, es preciso resaltar que en contextos interculturales, los programas académicos también ser acordes con las necesidades de desarrollo personal y social del niño. Esto se relaciona con la creación un espacio académico donde se establezca el diálogo intercultural a través del respeto hacia la diversidad y buscando objetivos en los que ellos se identifiquen. Por esto, es fundamental reconocer que las dificultades presentadas por los niños extranjeros en el aula se deben a que los contenidos y propósitos no les resultan significativos ni tampoco se ven reconocidos en estos (Rodríguez, 2003). Como consecuencia, el entorno escolar debe buscar la deconstrucción de las representaciones etnocéntricas de la cultura para generar un aprendizaje basado en la existencia compartida de representaciones culturales. Se trata ante todo de un proceso de descentración étnica para mejorar el conocimiento que los niños tienen de sí mismos y de la cultura del otro para mejorar la convivencia.

\section{Discusión y conclusiones}

Al analizar en qué medida influye la diversidad cultural en los procesos de integración entre los niños, se ha evidenciado que si bien la pertenencia a determinado grupo étnico o país puede influir 
en las relaciones entre los chicos, estos factores no siempre son un obstáculo. Esto se debe a que a pesar de pertenecer a determinado grupo social o cultural, los niños comportan consigo una identidad particular desarrollada a partir de sus procesos de socialización, su entorno familiar e incluso su trayectoria vital. Por tanto las relaciones que establecen con su entorno se vinculan con factores de carácter diverso. Efectivamente, se detectó que la mayor o menor capacidad de integración social entre los chicos, también está mediada por circunstancias relacionadas con el entorno familiar y social, así como la propia construcción de su identidad y las expectativas ante el futuro.

De igual manera se identificó que otro obstáculo surge cuando las instituciones y los educadores reconocen la importancia de favorecer la integración social y escolar de minorías étnicas e inmigrantes, pero no abordan adecuadamente dicha diversidad. De otro lado, cuando algunos reconocen estas carencias, no disponen de los recursos humanos o curriculares adecuados. Desde esta perspectiva, varios discursos detectados en esta investigación denotan una orientación asimilacionista. De este modo, el problema ni siquiera es la obvia necesidad de aprender la lengua y caracterizaciones propias de la sociedad de acogida, sino el énfasis que se pone en la normalización educativa y en la integración cultural como un asunto de los "otros" y no como un asunto de "todos".

Expuesto este argumento, queda claro que la integración social de los niños y jóvenes adquiere especial importancia porque de ello depende no solo su éxito académico sino también su desarrollo integral. Para ello, entonces es necesario replantear las estructuras curriculares a través de la reconstrucción de los contenidos haciendo énfasis en la interculturalidad. Ese planteamiento es imprescindible para comprender que la acogida inicial debe realizarse desde el principio partir de las interacciones entre compañeros y educadores autóctonos como elemento para lograr un proceso de inclusión efectivo. De hecho, para que esto se logre a cabalidad es necesario que los alumnos inmigrantes se integren en un contexto normalizado desde el primer momento ya que el desarrollo de su identidad y la construcción de sus procesos de socialización dependen del equilibrio en las relaciones dentro del aula.

Con respecto a este punto, es evidente que otro los aspectos vinculados con la integración, tiene que ver con el conjunto de actitudes que despliega la sociedad de acogida para atender la complejidad de procedencias culturales. En este contexto, resultan reveladoras las estrategias específicas para atender a las necesidades del niño en relación con su formación personal y académica desde la equidad en oportunidades y deberes. Desde luego, es imprescindible el reconocimiento de los derechos individuales y una práctica educativa que incluya también un compromiso de lucha contra la exclusión social para lograr un cambio real en la comunidad educativa (Montón, 2002). Efectivamente, una gestión efectiva de la interculturalidad, impulsando el conocimiento y la comprensión de la diversidad cultural en el ámbito educativo permite mejorar los procesos de integración social de los niños inmigrantes.

Por último, en el marco de los recientes procesos migratorios hacia Europa desde Siria y otros contextos de conflicto, es posible plantear que el desafío del contexto escolar consiste en incidir significativamente en el curso vital de los inmigrantes. Por ello, le corresponde al entorno educativo promover la construcción de relaciones interpersonales donde la diferencia no se convierta en sinónimo de antagonismo sino en posibilidad de construir y aprender colectivamente a partir del otro. En este sentido, el objetivo debe ser buscar el desarrollo integral del niño a través de una educación que fomente habilidades para la vida y capacidades para convivir en comunidad. Así, el reto de la escuela en contextos interculturales consiste en reorientar la trayectoria vital de niños que han dejado sus raíces, promoviendo un diálogo entre su pasado y su presente, permitiéndoles desarrollar estrategias concretas en favor de la construcción de su proyecto de vida en un nuevo contexto social. 


\section{Notas}

1. Programa orientado a fortalecer la inclusión social, las relaciones interculturales y la construcción del proyecto de vida de población inmigrante y gitana en España.

2. ONG orientada a fortalecer la inclusión social, laboral y educativa de población inmigrante y gitana en España.

3. Primera etapa de la educación básica obligatoria en España. Representada en las Gráficas 1 y 3 con la sigla de EPO.

4. Segunda y última etapa de la educación obligatoria en España antes de ingresar a la formación superior. Representada en las Gráficas 1 y 3 con la sigla de ESO.

\section{Referencias bibliográficas}

Aguado, M. T. (1991). La educación intercultural: Conceptos, paradigmas, realizaciones. En M. Jiménez Fernández (Ed.), Lecturas de pedagogía diferencial (pp. 89-104). Madrid: Dykinson.

Aguado, M. T. (2005). La educación intercultural en la práctica escolar: Investigación en el ámbito español. Revista de Educación, (7), 43-52.

Baczco, B. (1999). Los imaginarios sociales. Buenos Aires: Nueva Visión.

Banks, J. (1989). Multicultural education: Characteristics and goals. In J. Banks, \& C. McGee Banks (Eds.), Multicultural education: Issues and perspectives (pp. 20-26). Boston: Allyn and Bacon.

Blanco, M. (2006). La equidad y la inclusión social: Uno de los desafíos de la educación y la escuela hoy. REICE: Revista Electrónica Iberoamericana sobre Calidad, Eficacia $y$ Cambio en Educación, 4(3), 1-15.

Cabrera, F., Espín, J., Marin, M., Julia V., \& Rodríguez, M. (1998). Elaboración de un cuestionario para medir la identidad étnica y la aculturación en la adolescencia. Revista de Educación, (315), 227-249.

Carbonell, F. (1999). Desigualdad social, diversidad cultural y educación. En La inmigración extranjera en España: Los retos educativos (pp. 99-118). Barcelona: Fundación La Caixa.

Chartier, R.(1983). El mundo como representación. Historia cultural: Entre práctica y representación. Barcelona: Gedisa.

Consejo de Europa (1983). Resolución sobre la Cooperación Europea en Educación, adop- tada por la conferencia de Ministros de Educación, Dublín, 10 - 12 de mayo.

Colectivo Ioé (2005). Inmigrantes extranjeros en España: ¿reconfigurando la sociedad? Panorama Social, 1, 32-47.

Dávila, G. (2006). El razonamiento inductivo y deductivo dentro del proceso investigativo en ciencias experimentales y sociales. Laurus: Revista de Educación, 12, 180-205.

Dietz, G. (2003). Multiculturalismo, interculturalidad y educación: Una aproximación antropológica. Granada: Universidad de Granada.

Díaz, J., Franco, P., Martínez, L., \& Pozo, J. (2001). Inmigración y escuela. de la educación intercultural a la educación para la ciudadanía. Madrid: Ministerio de Trabajo y Asuntos Sociales - FETE-UGT.

Ley 36 / 2002, de 8 de octubre, de modificación del código civil en material de nacionalidad. Boletín Oficial del Estado, núm. 242, de 9 de octubre de 2002, pp. 35683 a 35640. Recuperado de https://goo.gl/35nTDK.

Monton, M. J. (2002). La educación del alumnado inmigrante. Un reto social y educativo. Anuario de Psicología, 33(4), 499-519.

Muñoz, A. (1997). Educación intercultural. Teoría y práctica. Madrid: Escuela Española.

Palaudarias, J. M. (1998). Inmigración, integración y escolarización. Revista Española de Educación Comparada, (4), 149-160.

Rodríguez, R. (2003). La atención a la diversidad cultural: Un reto educativo en la sociedad global: Algunas propuestas de intervención desde el enfoque intercultural. En Inmigración, interculturalidad y convivencia, 361-368. Ceuta: Instituto de Estudios Ceutíes.

Rodríguez, R. (2004). Atención a la diversidad cultural en la escuela. Propuestas de intervención socioeducativa. Educación y Futuro: Revista de Investigación Aplicada y Experiencias Educativas, 10, 37-47.

Sartori, G. (2001). La sociedad multiétnica. Pluralismo, multiculturalismo y extranjeros. Madrid: Taurus.

Vázquez, O. (2001). Acción intercultural y trabajo social. Cuadernos de Trabajo Social, (14), 29-43. 